\title{
Three-Dimensional Force Prediction of a Flexible Tactile Sensor Based on Radial Basis Function Neural Networks
}

\author{
Feilu Wang $\mathbb{D}^{1}$ and Yang Song $\mathbb{D}^{1,2}$ \\ ${ }^{1}$ School of Electronic and Information Engineering, Anhui Jianzhu University, Hefei 230601, China \\ ${ }^{2}$ Key Laboratory of Building Information Acquisition and Measurement Control Technology, Anhui Jianzhu University, \\ Hefei 230601, China
}

Correspondence should be addressed to Yang Song; esunny@ahjzu.edu.cn

Received 7 September 2020; Revised 24 January 2021; Accepted 5 February 2021; Published 17 March 2021

Academic Editor: Calogero M. Oddo

Copyright (C) 2021 Feilu Wang and Yang Song. This is an open access article distributed under the Creative Commons Attribution License, which permits unrestricted use, distribution, and reproduction in any medium, provided the original work is properly cited.

\begin{abstract}
A flexible tactile sensor array with $6 \times 6 \mathrm{~N}$-type sensitive elements made of conductive rubber is presented in this paper. The property and principle of the tactile sensor are analyzed in detail. Based on the piezoresistivity of conductive rubber, this paper takes full advantage of the nonlinear approximation ability of the radial basis function neural network (RBFNN) method to approach the high-dimensional mapping relation between the resistance values of the $\mathrm{N}$-type sensitive element and the threedimensional (3D) force and to accomplish the accurate prediction of the magnitude of 3D force loaded on the sensor. In the prediction process, the $k$-means algorithm and recursive least square (RLS) method are used to optimize the RBFNN, and the $k$ -fold cross-validation method is conducted to build the training set and testing set to improve the prediction precision of the 3D force. The optimized RBFNN with different spreads is used to verify its influence on the performance of 3D force prediction, and the results indicate that the spread value plays a very important role in the prediction process. Then, sliding window technology is introduced to build the RBFNN model. Experimental results show that setting the size of the sliding window appropriately can effectively reduce the prediction error of the $3 \mathrm{D}$ force exerted on the sensor and improve the performance of the RBFNN predictor, which means that the sliding window technology is very feasible and valid in 3D force prediction for the flexible tactile sensor. All of the results indicate that the optimized RBFNN with high robustness can be well applied to the 3D force prediction research of the flexible tactile sensor.
\end{abstract}

\section{Introduction}

With the rapid development of intelligent robot technology, researchers are eager to endow robots with a similar tactile perception of human skin to improve the robot's ability of man-machine interaction. Making quick response and correct decision according to the change of the outside world is an important challenge for the tactile sensor of intelligent robots [1]. Therefore, the research of flexible tactile sensors has become one of the hot topics in the intelligent robot skin field. The flexible tactile sensor refers to the sensor that not only has the characteristics similar to human skin but also can cover the robots' or other objects' surface, acting as electronic skin, sensing and measuring multidimensional tactile information. The flexible tactile sensor with high flexibility, sensitivity, extensibility, and ability of perceiving multidimensional tactile signals plays an irreplaceable role in the study of intelligent robots.

The flexible tactile sensor is mainly developed based on the principle of capacitance [2-5], piezoelectric effect [6-9], and piezoresistive effect $[10,11]$. In order to make the tactile sensor similar to human skin, more and more researchers are dedicating to the research of flexible tactile sensors with different functions and different materials. Massari et al. [12] presented the model based on numerical FEM for the development and calibration of a soft tactile sensor able to solve both the magnitude and the position of an applied normal load on its surface, which is successfully validated through experimental results. Schwartz et al. [13] reported the fabrication of flexible pressure-sensitive organic thin- 
film transistors, which demonstrated that their sensors can be used for noninvasive, high-fidelity, continuous radial artery pulse wave monitoring, which may lead to the use of flexible pressure sensors in mobile health monitoring. Asadnia et al. [14] presented a highly stretchable, self-powered, and ultrasensitive strain sensor based on piezoelectric PVDF nanofibers. Lee et al. [15] illustrated a flexible capacitive tactile sensor array with the capability of measuring both the normal and shear force distributions using PDMS as a base material, which is realized in an $8 \times 8$ sensor array, and each unit responds to normal and shear stresses. Based on a $3 \times 3$ sparse flexible tactile sensor array, Liu et al. [16] realized the detection of arbitrary contact force loaded on the sparse tactile sensor by utilizing the inverse solution method and the diffusion effect of the elastomer cover. Mittendorer et al. [17] proposed a novel approach to realize whole-body tactile interactions with self-organizing, multimodal artificial skin on a robot. Cirillo et al. [18] exploited the perception data provided by a tactile sensor to obtain normal and tangential components of the contact force and proposed an algorithm to extract useful information from tactile raw data.

Due to the particularity of the sensor structure and manufacturing process, the research on flexible tactile sensors concentrates on material development, structure design, and sensor array size. As to the $3 \mathrm{D}$ force detection of flexible tactile sensors, it is difficult to predict and recognize the $3 \mathrm{D}$ force from different contact spots. There inevitably exist errors in the process of tactile perception and $3 \mathrm{D}$ force detection of the flexible tactile sensor. Therefore, precise detection and prediction of contact force are particularly important in the research of flexible tactile sensors.

In this paper, a flexible tactile sensor array based on the conductive rubber with piezoresistivity is studied. Its principle and mathematical model are analyzed in detail, the relationship between the resistance vector and the $3 \mathrm{D}$ force vector is approximated, and it realizes the accurate prediction of multiple $3 \mathrm{D}$ forces exerted on the sensor by the radial basis function neural network (RBFNN) intelligent algorithm. This paper is organized as follows. Section 2 introduces the property, the structure, and the principle of the flexible tactile sensor. Section 3 demonstrates the prediction process of $3 \mathrm{D}$ forces based on the RBFNN from different aspects and perspectives. Section 4 presents the conclusion.

\section{Property and Principle of the Flexible Tactile Sensor}

2.1. Characteristics of Conductive Rubber. In this paper, the conductive rubber is used as the main material of the sensitive element for the flexible tactile sensor, which is mainly composed of the GD-401 silicon rubber that is evenly filled by the CB3100 carbon black conductive particle with a volume fraction of $28 \%$. The resistivity of the carbon black is 8 $\times 10^{-3} \Omega \cdot \mathrm{cm}$. Young's modulus, Poisson's ratio, bulk modulus, and shear modulus of the conductive rubber are $6.1 \mathrm{MPa}$, $0.49,101.67 \mathrm{MPa}$, and 2.05 $\mathrm{MPa}$, respectively. The conductive rubber not only has the flexibility of rubber but also has great conductivity. When it is subjected to the external force, it would be deformed at that moment, and the relative concen- tration of the conductive particles inside the conductive rubber would be changed accordingly, which will lead to the change of resistivity. When pressure is loaded, the gaps among conductive particles become smaller and the concentration increased, which generates a low resistivity. In the case of no load exerted on the conductive rubber, there are larger gaps among the conductive particles and the concentration of conductive particles decreased, which makes a high resistivity. That phenomenon is called piezoresistivity. The conductive rubber is very sensitive to tactile force and stress, and the corresponding deformation of the conductive rubber is closely related to the stress [19]. In view of its excellent properties, conductive rubber has been widely used in the research of flexible tactile sensors $[19,20]$. Based on the characteristics of conductive rubber, the $3 \mathrm{D}$ force of the flexible tactile sensor is precisely predicted by the RBFNN method in this paper.

2.2. Model of the Sensor. In order to develop the sensing ability of the flexible tactile sensor and the prediction accuracy of $3 \mathrm{D}$ force, this paper carries out a comprehensive study on the model of the flexible tactile sensor array which contains $36 \mathrm{~N}$-type sensitive elements as shown in Figure 1. The N-type sensitive element is very sensitive to the tactile force and stress, and it is composed of three conductive columns (labeled " 1 ," " 2 ," and " 3 ") which are made of the conductive rubber. It can decompose $3 \mathrm{D}$ force into three components and transmit the force components to the three conductive columns.

The sensor array model shown in Figure 1 is mainly composed of force-sensitive material. It includes three parts: the sensing array is composed of $36 \mathrm{~N}$-type tactile forcesensitive elements made of the conductive rubber; the frame of the array is made of flexible insulating GD-401 silicon rubber, and the interspaces among the $\mathrm{N}$-type sensitive elements are filled with the silicon rubber; and the wires connect to the external circuit, and the N-type sensitive elements are connected by the wires. The resistance of the three conductive columns of every sensitive element could be obtained by detecting the resistances between the upper wire and the corresponding lower wire. For example, the resistance of conductive column " 3 " of the N-type sensitive element located at the top right corner in Figure 1 can be gained by detecting the upper wire labeled " 8 " and the lower wire labeled " 9 ." The initial length of the conductive columns labeled " 1 ," " 2 ," and " 3 " are $\sqrt{2} \mathrm{~cm}, 1 \mathrm{~cm}$, and $\sqrt{2} \mathrm{~cm}$. The angles between columns " 1 " and " 2 " and between columns " 2 " and " 3 " are both $45^{\circ}$. The cross-sectional area of each conductive column is $4 \mathrm{~mm}^{2}$. The size of the $6 \times 6$ sensitive element array shown in Figure 1 is $12 \mathrm{~cm} \times 12 \mathrm{~cm} \times 1 \mathrm{~cm}$.

When the load is applied on the sensor, the resistances of the corresponding conductive columns would be changed subtly. As the lower surface is usually attached or fixed on the robot or other objects, it is supposed that the electrodes on the lower surface have no displacement. In view of the above, the prototype of the flexible tactile sensor based on the conductive rubber is produced. The model of the tactile sensor is drawn on SolidWorks 2014 (SolidWorks Inc., Waltham, USA). The 3D printing technology is conducted to 


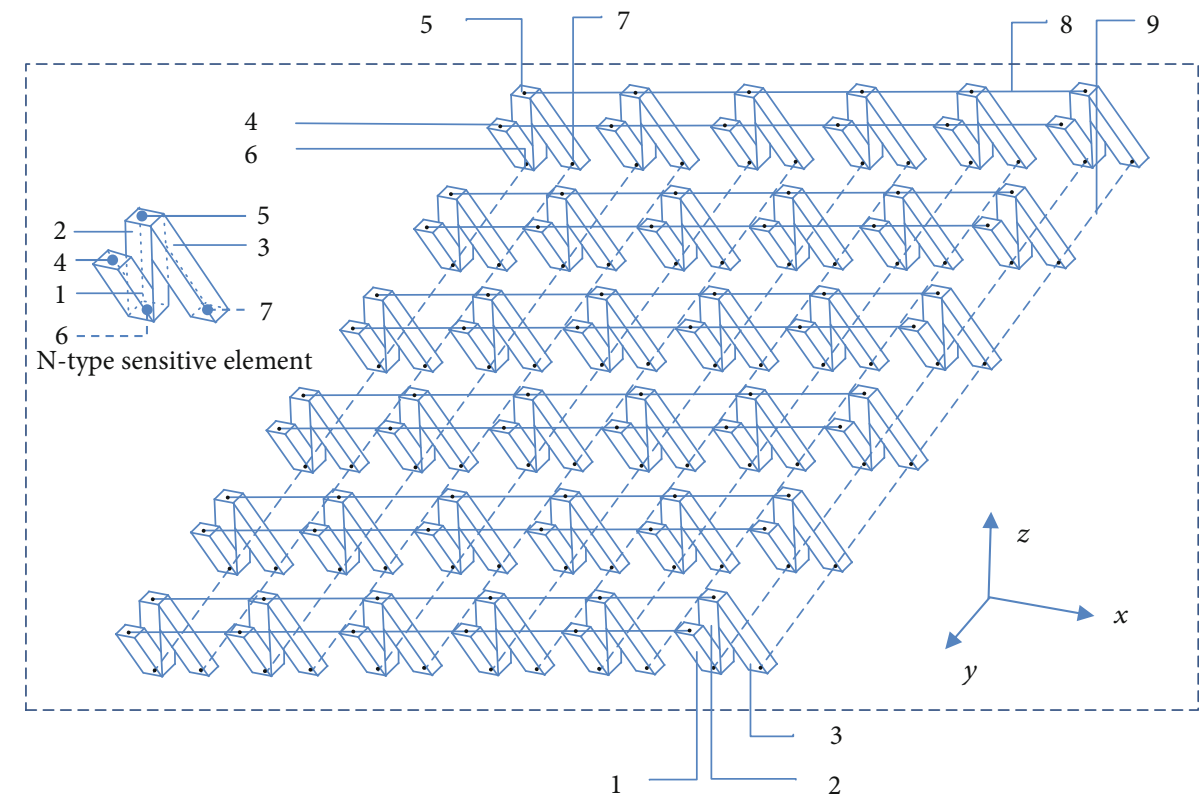

Figure 1: Model of the flexible tactile sensor array. "1," "2," and " 3 " denote the three conductive columns of the N-type sensitive element; " 4 " and " 5 " denote the electrodes on the upper surfaces of the conductive columns; " 6 " and " 7 " denote the electrodes on the lower surfaces of the conductive columns; "8" and " 9 " denote the upper wires and the lower wires that connect the electrodes. Each upper wire is perpendicular to the lower wires.

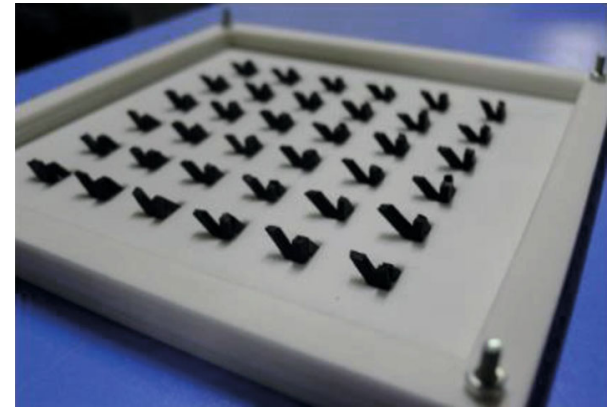

FIgURE 2: 3D printed mold with $6 \times 6$ sensitive elements.

print the mold for the $6 \times 6$ sensor array model, and the 36 $\mathrm{N}$-type force-sensitive elements made by the conductive rubber are placed into the corresponding holes of the mold, as shown in Figure 2 [11]. After that, the insulating GD-401 silicon rubber filler is poured into the gaps among the sensitive elements, and it is important to notice that the electrodes should not be covered by the insulating rubber. Then, the upper wires and the lower wires are arranged according to the distribution as in Figure 1. Meanwhile, the wires are sealed in the insulating rubber. Finally, the entity of the flexible tactile sensor based on the conductive rubber and the silicon rubber is generated, as shown in Figure 3.

2.3. Theoretical Analysis. It is on the assumption that the sensitive element is a continuous, isotropic, and nonhysteretic elastomer [21], and the volume of the conductive column remains unchanged when it is subjected to a suitable force. The resistance values of the conductive columns satisfy the following rules:

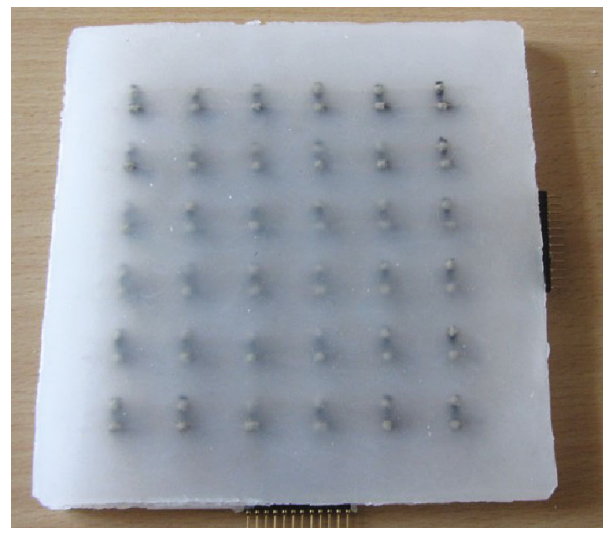

Figure 3: Prototype of the flexible tactile sensor.

$$
\begin{aligned}
R_{i} & =\rho \frac{l_{i}}{S}=\rho \frac{l_{i}^{2}}{V_{i}}, \quad i=x, y, z, \\
l_{i} & =\sqrt{\frac{R_{i} \bullet V_{i}}{\rho}}, \quad i=x, y, z, \\
\Delta l_{i} & =\left|l_{i}^{\prime}-l_{i}\right|=\sqrt{\frac{V_{i}}{\rho}} \cdot\left|\sqrt{R_{i}^{\prime}}-\sqrt{R_{i}}\right|, \quad i=x, y, z,
\end{aligned}
$$

where $R_{x}, R_{y}$, and $R_{z}$ represent the resistance of the corresponding conductive column labeled " 3 ," " 1 ," and " 2 "; $R_{x}^{\prime}$, $R_{y}^{\prime}$, and $R_{z}^{\prime}$ represent the resistance of the conductive column labeled " 3 ," "1," and " 2 " when the force is loaded on the sensitive element; $l_{x}, l_{y}$, and $l_{z}$ represent the original length of the 


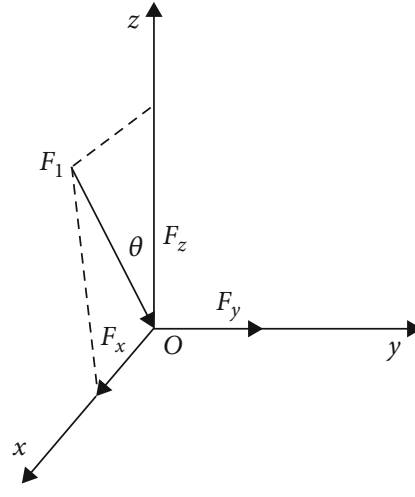

(a) Force analysis diagram of $F_{1}$

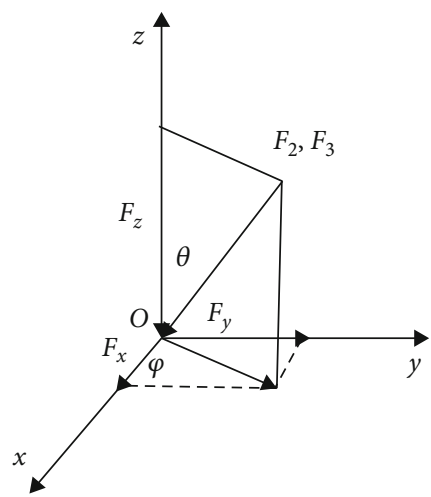

(b) Force analysis diagram of $F_{2}$ and $F_{3}$

FIGURE 4: Force analysis diagram.

corresponding conductive column labeled " 3 ," "1," and "2"; $l_{x}^{\prime}, l_{y}^{\prime}$, and $l_{z}^{\prime}$ represent the length of the conductive column labeled " 3 ," "1," and " 2 " when the force is loaded; $\Delta l_{x}, \Delta l_{y}$, and $\Delta l_{z}$ represent the change in length of the conductive column labeled " 3 ," "1," and "2"; $\rho$ is the resistivity; $V_{x}, V_{y}$, and $V_{z}$ represent the volume of the corresponding conductive column labeled "3," "1," and "2"; $S$ represents the crosssectional area of the conductive column.

As an elastomer, the conductive column satisfies the general Hooke's law and the relationship between stress and strain is linear.

$$
\sigma=E \bullet \varepsilon=E \bullet \frac{\Delta l_{i}}{l_{i}}=\frac{F_{i}}{S}, \quad i=x, y, z
$$

where $\sigma, \varepsilon$, and $E$ denote stress, strain, and Young's modulus, respectively. follows:

Combining expressions (3) and (4), it could be gained as

$$
\Delta l_{i}=\frac{F_{i} \bullet l_{i}}{E \bullet S}, \quad i=x, y, z
$$

Then, the approximate relation between the 3D force and the resistance of the conductive column could be computed as

$$
F_{i}=\left(\frac{E \cdot S}{l_{i}}\right) \cdot \sqrt{\frac{V_{i}}{\rho}} \cdot\left|\sqrt{R_{i}^{\prime}}-\sqrt{R_{i}}\right|, \quad i=x, y, z .
$$

It is generalized that

$$
F_{i}=f_{k}\left(R_{i}^{\prime}\right)=f_{k}\left(\Delta R_{i}\right)=g_{k}\left(\Delta l_{i}\right), \quad i=x, y, z ; k=1,2,3,
$$

where $f_{k}$ is the function that shows the relation between $R_{i}^{\prime}$ and $F_{i}$, and $g_{k}$ is the function that shows the relation between $\Delta l_{i}$ and $F_{i}$.
The flexible tactile sensor realizes the decomposition of three-dimensional information, avoids the direct interference between sensitive elements, reduces the complexity of the sensor, and accelerates the pace of real-time prediction of $3 \mathrm{D}$ force loaded on the sensor.

As the length of the conductive column is very short, the magnitude of deformation is also small, which does not exceed $20 \%$ of its own length. It can be considered that $\Delta l_{i} \cong \Delta i(i=x, y, z)$, where $\Delta i$ represents the displacement of the electrode along the $i$-direction. If $\Delta l_{i}$ changed under pressure, then the value of $R_{i}$ would be changed correspondingly, and the force $F_{i}$ loaded on the sensor can be solved by detecting the corresponding resistance signal $R_{i}^{\prime}$.

When the 3D force is conducted to the sensor, it can be decomposed into three components by the three conductive columns. On the basis of the status equation (7), the force components could be figured out. The analysis diagram of the sensitive element under force is shown in Figure 4.

The decomposition of the 3D force for the flexible sensor is as follows:

$$
\begin{aligned}
& F_{x}=f_{1}\left(F_{2} \cos \varphi+F_{3} \cos \varphi-F_{1} \sin \theta\right), \\
& F_{y}=f_{2}\left(F_{2} \sin \varphi-F_{3} \sin \varphi\right), \\
& F_{z}=f_{3}\left(F_{1} \cos \varphi+F_{2} \cos \varphi+F_{3} \cos \theta\right) .
\end{aligned}
$$

$F_{x}$ and $F_{y}$ represent the shear force components along the $x$-axis and $y$-axis. $F_{z}$ represents the normal force component along the $z$-axis. $F_{1}, F_{2}$, and $F_{3}$ are the external forces exerted on the sensor. $\varphi$ denotes the angle between the projections of $F_{2}$ or $F_{3}$ on the $x-O-y$ plane and the $x$-axis, and $\theta$ is the angle between $F_{j}(j=1,2,3)$ and $z$-axis.

With positions and coordinates of the upper electrodes on the sensitive elements obtained, the deformation status of the flexible tactile sensor with stress could be acquired accurately. The stress applied on the surface of the sensor could be exactly gained by the spatial positions and deformation of several discrete points.

The potential theory [22] had solved the problem of how to obtain the deformation of the elastomer by loading $3 \mathrm{D}$ 


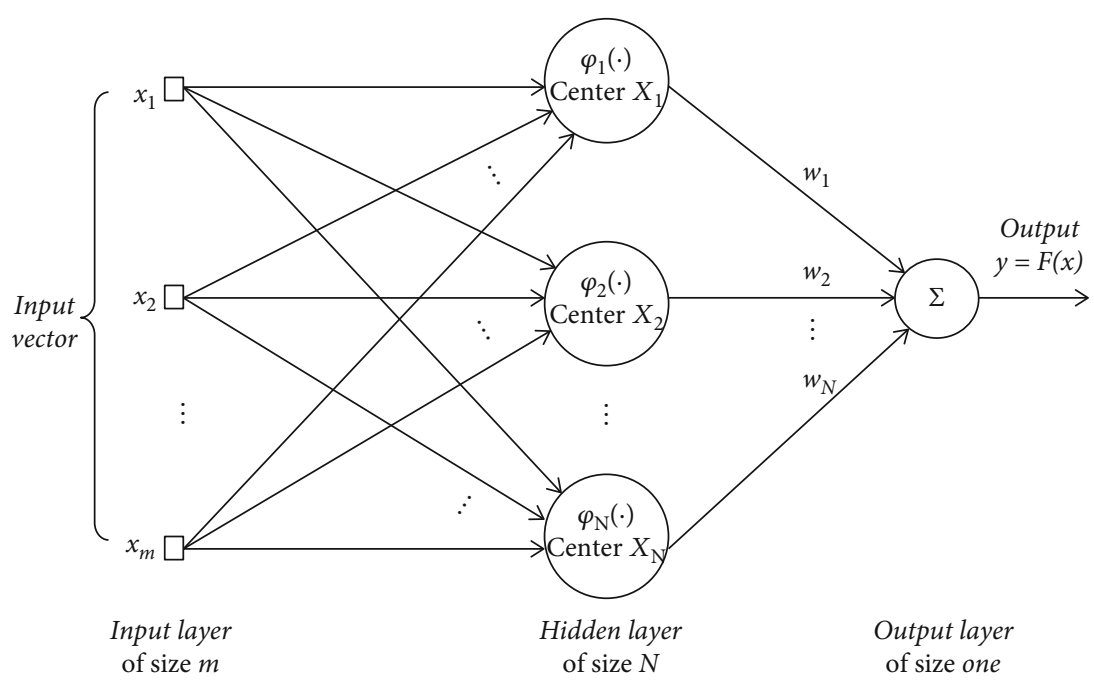

FIGURE 5: The structure of RBFNN.

forces on the elastic semispace. That is also applicable to the flexible tactile sensor studied in this paper. The potential theory [22] indicates that the displacement of the point far from the loading region is reduced by $1 / \rho . \rho$ is the distance between the point on the loading region and the point inside the elastomer. That means the points with a large distance from the loading region almost have no elastic displacement. As the displacements have been found, the stresses are calculated from the corresponding strains by Hooke's law.

According to the principle of superposition [23], it implies that the mathematical relation between the $3 \mathrm{D}$ force loaded on and the deformation of the elastomer can be linearly superimposed. It provides a novel idea to solve the $3 \mathrm{D}$ force-deformation problem. In practice, the normal force and tangential force could be studied, respectively, and the deformation of the elastomer caused by the 3D force could be obtained by synthesizing the relevant conclusions according to the superposition principle.

The above denotes that with the $3 \mathrm{D}$ force components exerted on, the displacement along the $i$-direction $(i=x, y, z$ ) can be gained. According to the superposition theory [23], the tangential displacement and the normal displacement from a point of the elastomer are the integration of the displacement generated by each force loading at that point. In view of the principles above, this paper focuses on predicting the multiple 3D force loaded on the sensor with high precision and approaching the high-dimensional mapping between the resistance vector and the 3D force vector based on the optimized RBFNN.

\section{Prediction of 3D Force Based on the RBFNN}

Based on the piezoresistive effect of conductive rubber, this paper takes full advantage of the approximation ability of the RBFNN method to accomplish the accurate prediction of the magnitude of 3D force for the flexible tactile sensor and approach the high-dimensional mapping relation between the resistance values of the $\mathrm{N}$-type sensitive element and the $3 \mathrm{D}$ force.
3.1. The RBFNN Method. The RBFNN is widely concerned by researchers because of its simple structure and great generalization ability. It had been proved that RBFNN can approximate any nonlinear function with arbitrary precision [24, 25]. RBFNN is a three-layer forward network, as shown in Figure 5, and each layer is composed of neuron nodes. The first layer is the input layer which consists of the original signal nodes. The second layer is the hidden layer (it is also called radial basis layer) whose activation function is a locally distributed nonnegative and nonlinear function, and the function is radially symmetric and gradually attenuated according to the center point. The third layer is the output layer; the linear weighted sum of the output of the hidden layer neurons constitutes the output of the RBF network.

The input layer of RBFNN is composed of $m$ neuron nodes. In general, the number of neuron nodes in the hidden layer is equal to the number of training samples. The hidden layer adopts the radial basis function as the activation function; then, the corresponding output of the neuron nodes in the hidden layer is computed in (9). In this paper, the Gaussian function is taken as the radial basis function.

$$
\begin{aligned}
\varphi_{j}(X) & =\varphi\left(\| X-X_{j}||\right) \\
& =\exp \left(-\frac{1}{2 \sigma_{j}^{2}}\left\|X-X_{j}\right\|\right), \quad j=1,2, \cdots, N,
\end{aligned}
$$

where vector $X$ is the input layer signal, $X_{j}$ is the center of the radial basis function (Gaussian function), $\sigma_{j}$ represents the spread width of the Gaussian function of the center $X_{j}, N$ is the number of hidden layer nodes, and $\varphi(\bullet)$ is the activation function of the hidden layer. In this paper, $m$ is 36 , and $N$ is 2700 which is the number of the training samples.

Each neuron node in the input layer is directly connected to all the neuron nodes in the hidden layer, and every node in the hidden layer is connected to all the neuron nodes in the output layer by the weights, respectively. The RBFNN could 
accomplish the global approximation of the objective function and could accurately fit the training data.

The activation function of the output layer is linear, and the output is shown in (10)

$$
F\left(X_{i}\right)=\sum_{j=1}^{\mathrm{N}} \omega_{j} \varphi\left(\left\|X_{i}-X_{j}\right\|\right)=d_{i}, \quad i, j=1,2, N
$$

where $\omega_{j}$ is the weight between the $j$-th neuron of the hidden layer and the neuron of the output layer, $X_{i}$ is the input signal/vector of the input layer, $X_{j}$ is the center of the radial basis function, and $d_{i}$ is the expected output according to the input signal $X_{i}$.

Let $\varnothing=\left\{\varphi\left(\left\|X_{i}-X_{j}\right\|\right)\right\}_{i, j=1}^{N}, w=\left[\omega_{1}, \omega_{2}, \cdots, \omega_{N}\right]^{T}$, and $d=\left[d_{1}, d_{2}, \cdots, d_{N}\right]^{T} . w$ and $d$ represent the weight coefficient vector and the expected output, respectively. Then, equation (10) could be substituted as follow:

$$
\varnothing w=d
$$

If each $X_{i}(i=1,2, N)$ is different and $\varnothing$ is reversible, then $w$ can be figured out by (12)

$$
w=\varnothing^{-1} d
$$

The Gaussian function satisfies the Micchelli theorem. According to the Micchelli theorem, all the input vectors are different, which ensures that the matrix $\varnothing$ is reversible. It indicates that the weight vector $w$ could be correctly solved.

Two of the key problems in building the RBFNN model are how to set the center for the Gaussian function of the hidden layer and how to figure out the weights between the hidden layer and the output layer. In the experiment, the $k$ -means clustering algorithm and recursive least square (RLS) method are used to optimize RBFNN and solve the two problems, respectively.

In this paper, the RBFNN is used as a predictor to predict the magnitude of 3D force applied on the flexible tactile sensor. As the relation between the input layer and the hidden layer is nonlinear and the relation between the hidden layer and the output layer is linear, in the prediction process, the Gaussian function and linear function are used as the activation function of the hidden layer and the output layer, separately. The input vector of the RBFNN is an $m$-dimensional vector that consisted of resistances of the conductive columns, and the output vector is an $n$-dimensional vector that consists of force components loaded on the sensor. The training samples and testing samples for the $3 \mathrm{D}$ force prediction are constructed by the $k$-fold cross-validation method.

3.2. Prediction of 3D Force with Different Spreads. When the external force is applied on the upper surface of the flexible tactile sensor, the $\mathrm{N}$-type sensitive elements in the array would be deformed, and the resistance values of the conductive columns would be changed accordingly. The resistance can be obtained by scanning the circuit. Due to the special model of the sensitive element, the 3D force could be decomposed into three components, which are the normal force $F_{z}$ and the tangential forces $F_{x}$ and $F_{y}$. The RBFNN is constructed to predict the $3 \mathrm{D}$ forces received by the sensor array, and the relationship between the resistance vector and the force vector is decomposed and approached.

In the experiment, 3000 samples are collected; each sample includes the 36-dimensional resistance vector and the corresponding 36-dimensional force vector. In terms of the normal force $F_{z}$, the input layer of the RBFNN is composed of the 36-dimensional resistance vector $R_{z}=\left[R_{z}^{1}, \cdots, R_{z}^{i}, \cdots\right.$, $\left.R_{z}^{36}\right]$, where $R_{z} \in[16 \mathrm{k} \Omega, 25 \mathrm{k} \Omega] . R_{z}^{i}$ represents the column resistance labeled " 2 " of the $i$-th sensitive element in the sensor array. Correspondingly, the output layer of the RBFNN is composed of the 36-dimensional force vector $F_{z}=\left[F_{z}^{1}, \cdots\right.$, $\left.F_{z}^{i}, \cdots, F_{z}^{36}\right]$, where $F_{z}^{i} \in[0 \mathrm{~N}, 4.8 \mathrm{~N}] . F_{z}^{i}$ represents the normal force applied to the $i$-th sensitive element. According to the tangential forces $F_{x}$ and $F_{y}$, the input layer is composed of the 36-dimensional resistance vectors $R_{x}=\left[R_{x}^{1}, \cdots, R_{x}^{i}, \cdots\right.$, $\left.R_{x}^{36}\right]$ and $R_{y}=\left[R_{y}^{1}, \cdots, R_{y}^{i}, \cdots, R_{y}^{36}\right]$, respectively, where $R_{x}^{i}, R_{y}^{i}$ $\in[32 \mathrm{k} \Omega, 50 \mathrm{k} \Omega] . R_{x}^{i}$ and $R_{y}^{i}$ represent the column resistances labeled " 3 " and " 1 " of the $i$-th sensitive element in the sensor array, respectively. The output layer of the RBFNN is composed of the 36-dimensional force vectors $F_{x}=\left[F_{x}^{1}, \cdots, F_{x}^{i}\right.$, $\left.\cdots, F_{x}^{36}\right]$ and $F_{y}=\left[F_{y}^{1}, \cdots, F_{y}^{i}, \cdots, F_{y}^{36}\right]$, respectively, where $F_{x}^{i}$, $F_{y}^{i} \in[0 \mathrm{~N}, 4.8 \mathrm{~N}] . F_{x}^{i}$ and $F_{y}^{i}$ represent the tangential forces applied to the $i$-th sensitive element.

In the initial dataset, the magnitude of resistance is much wider than that of $3 \mathrm{D}$ force, which would impact the prediction accuracy of force and reduce the approximation capability of the RBFNN predictor. So, the input values would be normalized, and the min-max normalization formula shown in (13) is used to transform the magnitude of resistance into $[16,25]$ or $[32,50]$, correspondingly.

$$
v^{\prime}=\frac{v-\min }{\max -\min }\left(\text { new }_{\max }-\text { new }_{\min }\right)+\text { new }_{\min },
$$

where $v$ is the initial value, and min and max are the lower bound and upper bound of the initial values, respectively. The new_ max and new_ min are the new lower bound and new upper bound, respectively.

In the process of constructing the RBFNN predictor, the $k$-fold cross-validation method is conducted to build the training set and testing set. 3000 samples are divided into 10 groups, which means that each group has 300 samples. Each time, 9 groups are selected as the training set and the one left is taken as the testing set, and that process is repeated 10 times. The prediction accuracy of 3D force is the average result of the $k$-fold cross-validation method based on the RBFNN. Meanwhile, the $k$-means algorithm and RLS method are used to optimize the RBFNN. The RBFNN is trained by using the training set to approximate the relationship between the resistance of the sensitive element and the force applied on the sensor array. Then, the RBFNN predictor model is constructed. After that, the RBFNN model is used to predict the force of the testing set, and the prediction 
error of the 3D force is measured by the average value of the 10 times prediction results based on the $k$-fold crossvalidation method.

During the experiment, the RBFNN model is built with different spread values. The spread value is very important for the RBFNN. The larger the spread of the radial basis function is, the smoother the fitting of the function would be, but a large spread value means that a very large number of neurons are needed to adapt to the rapid changes of the function. If the spread is too small, it means that many neurons are needed to adapt to the slow change of the function. If the spread is not set properly, it would lead to poor network performance. In this paper, the Gaussian function is used as the activation function of the hidden layer. The RBFNN based on the $k$-fold cross-validation method is constructed with different spread values (from 1 to 10 ) to verify its influence on the performance of $3 \mathrm{D}$ force prediction, and the prediction results of $F_{x}, F_{y}$, and $F_{z}$ for the flexible tactile sensor based on the RBFNN are shown in Table 1. The results in Table 1 are the mean relative errors between the actual force components exerted on the sensor and the force components predicted by the RBFNN model. The results indicate that the RBFNN can precisely approximate the high-dimensional nonlinear mapping between the resistance and the $3 \mathrm{D}$ force, and it has strong robustness and antinoise capability.

$$
E_{F i}=\frac{\sum_{t=1}^{s * 36}\left|\left(F_{i_{t}}^{\prime}-F_{i_{t}}\right) / F_{i_{t}}\right|}{(s * 36)}, \quad i=x, y, z ; t=1,2, \ldots s * 36 .
$$

In (14), $s$ is the amount of testing samples of the sensor array, $E_{F i}$ denotes the mean relative prediction error of force component $F_{i}(i=x, y, z)$ for $300(s=300)$ testing samples. $F_{i_{+}}$represents force component $F_{i} \quad(i=x, y, z)$ applied on the sensor of the $((t-1) \bmod 36+1)$-th sensitive element of the $((\mathrm{t}-1) / 36+1)$-th testing sample, and $F_{i_{t}}^{\prime}$ represents the prediction results of $F_{i_{t}}$ based on the RBFNN.

It can be seen from Table 1 that, with the increase of the spread value, the prediction error of the 3D force component presents a significant downward trend firstly. In particular, when the spread value is 7 , the optimal mean relative prediction errors of the 3D forces are obtained, which are $E_{F z}=$ $1.82 \%, E_{F x}=2.62 \%$, and $E_{F y}=3.44 \%$. After that, the prediction errors tend to rise up with the increase of the spread value. It indicates that spread plays an important role in the RBFNN, and its value is not the larger the better. The spread value should be properly set to gain the best result, and in this experiment, 7 is the optimal spread value.

The results in Table 1 reflect that the RBFNN model has great prediction ability and performance in approaching the high-dimensional nonlinear mapping relationship between the resistance vector and the force vector. The RBFNN constructed in this paper can be well applied to the $3 \mathrm{D}$ force prediction research of the flexible tactile sensor.

In order to show the results exactly, the prediction results of 180 force components, including 5 testing samples each
TABle 1: Prediction results of force components with different spreads.

\begin{tabular}{lccc}
\hline \multirow{2}{*}{ Spread } & \multicolumn{3}{c}{ Prediction error of 3D force romponents } \\
& $E_{F x}$ & $E_{F y}$ & $E_{F z}$ \\
\hline 1 & $6.34 \%$ & $7.29 \%$ & $4.73 \%$ \\
2 & $4.29 \%$ & $5.37 \%$ & $3.62 \%$ \\
3 & $3.87 \%$ & $4.76 \%$ & $4.48 \%$ \\
4 & $3.76 \%$ & $5.09 \%$ & $3.51 \%$ \\
5 & $3.97 \%$ & $4.65 \%$ & $3.01 \%$ \\
6 & $3.06 \%$ & $3.94 \%$ & $2.35 \%$ \\
7 & $2.62 \%$ & $3.44 \%$ & $1.82 \%$ \\
8 & $2.95 \%$ & $3.02 \%$ & $2.57 \%$ \\
9 & $3.64 \%$ & $3.67 \%$ & $3.21 \%$ \\
10 & $3.87 \%$ & $4.35 \%$ & $3.16 \%$ \\
Average error & $3.84 \%$ & $4.56 \%$ & $3.25 \%$ \\
\hline
\end{tabular}

consisting of 36 components $F_{i}(i=x, y, z)$, are randomly extracted from the testing set. The prediction results of $F_{x}$, $F_{y}$, and $F_{z}$ based on RBFNN with spread $=7$ are shown in Figures $6-8$, respectively. In Figures $6-8$, the blue circle represents the force component $F_{i}(i=x, y, z)$ along the $i$ -direction predicted by the RBFNN, the red star represents the actual force $F_{i}(i=x, y, z)$ along the $i$-direction applied on the sensitive element, and the black circle represents the absolute error between actual force $F_{i}$ and corresponding predicted $F_{i}(i=x, y, z)$. The mean relative prediction errors of $F_{x}, F_{y}$, and $F_{z}$ in Figures 6-8 for the 180 force components are $3.40 \%, 4.61 \%$, and $1.96 \%$, and the corresponding standard deviations of the relative errors of $F_{x}, F_{y}$, and $F_{z}$ are $0.2247,0.0901$, and 0.1862 , respectively.

As shown in Figures 6-8, when spread $=7$, the absolute prediction errors of $3 \mathrm{D}$ force components based on the RBFNN are very small, which can meet the practical application requirements. In particular, in Figure 8, the prediction results of $F_{z}$ are almost equal to the actual $F_{z}$, which means that the normal force $F_{z}$ could be well sensed by the sensitive elements with high precision. And by the special structure of the sensitive element, there exists a weak correlation between $F_{z}$ and other force components. As there exists strong coupling relation between $F_{x}$ and $F_{y}$, their prediction results shown in Figures 6 and 7 are slightly worse than those of $F_{z}$. The absolute prediction errors of $F_{x}, F_{y}$, and $F_{z}$ in Figures 6-8 for the 180 force components are shown in Figure 9.

The minimum absolute error, maximum absolute error, mean absolute error, and standard deviation of the absolute errors for the 180 force components $F_{i}(i=x, y, z)$ are shown in Table 2. Table 2 shows that the method proposed in the paper is suitable for the $3 \mathrm{D}$ force prediction, especially for the normal force.

3.3. Prediction of $3 D$ Force Based on Sliding Window Technology. It is found that when the external force is loaded on a sensitive element, other sensitive elements around it would be affected by its impact and get deformed. The 


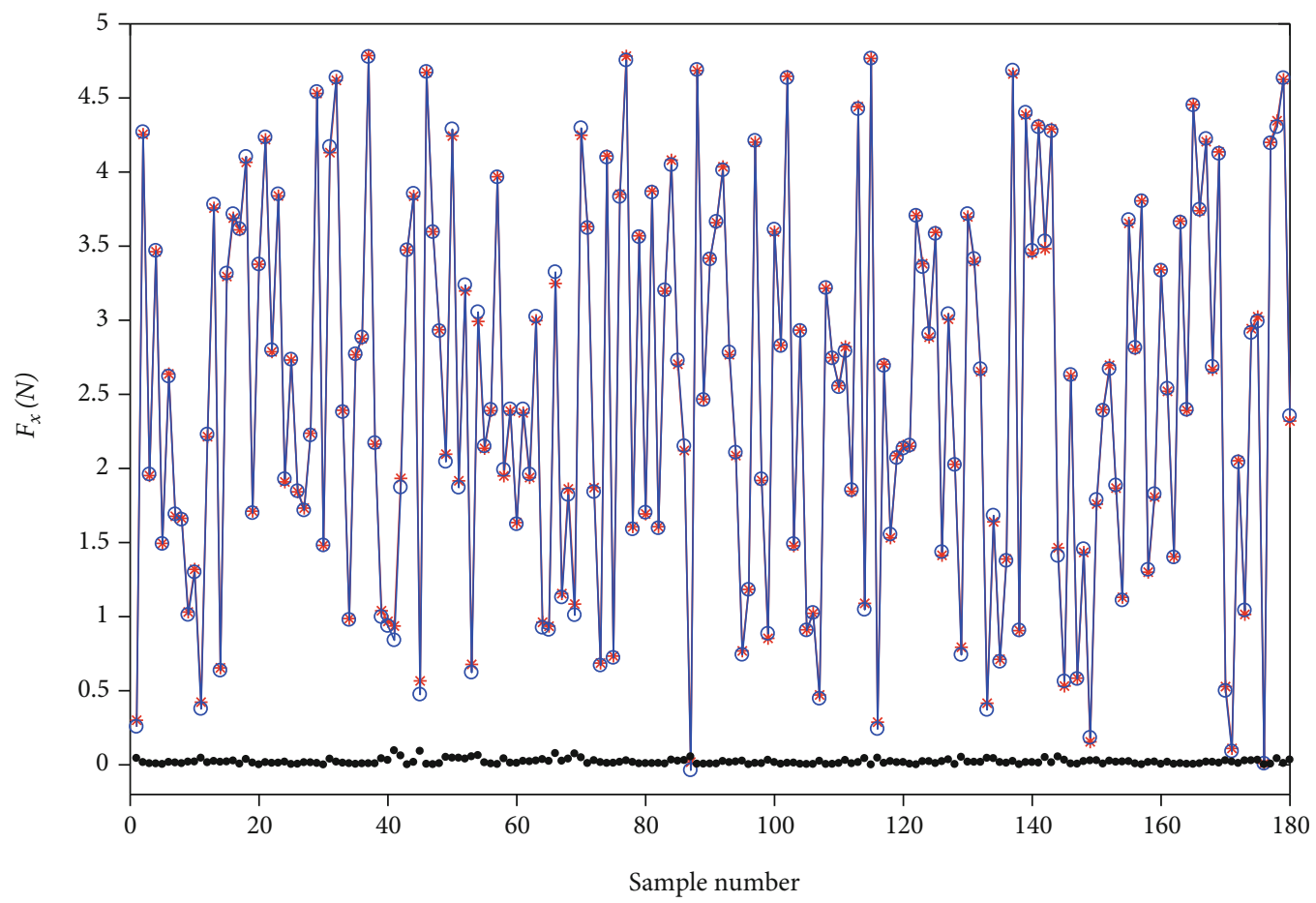

* Actual $F_{x}$
- Predicted $F_{x}$
- Absolute error of $F_{x}$

FIGURE 6: Prediction results of $F_{x}$ (the mean relative prediction error is 3.4\%).

experiments found that only the sensitive element within $1 \mathrm{~cm} \sim 2 \mathrm{~cm}$ distance around the force point could be significantly affected. If the sensitive element is far from the force point, it is hardly affected by its influence.

When a sensitive element senses the force loaded, the resistances of its three conductive columns could also be affected by the deformation status of its neighbor sensitive elements. In order to verify how the feature information of adjacent sensitive elements impact the target sensitive element, "sliding window" technology is introduced when constructing the input layer of the RBF network. The sliding window consists of the characteristic information of sensitive elements close to the target sensitive element and the information of the target element itself, which form the input layer vector together for the RBFNN. In the experiment, resistance is used as the characteristic information. Resistance values from 2,4 , and 6 sensitive elements closest to the target sensitive element are extracted to build the input layer vector, respectively. Therefore, the sliding window sizes of the input vectors are 3,5, and 7, respectively, as it includes 3,5 , and 7 sensitive elements' resistance information, and each sensitive element consists of 3 columns. Correspondingly, the input layer vector of the new RBFNN is the resistance with $36 \times 3$ $\times 3$ dimensions, $36 \times 5 \times 3$ dimensions, and $36 \times 7 \times 3$ dimensions, separately, and the corresponding output layer is a $3 \mathrm{D}$ force vector with $36 \times 3$ dimensions.

During the training procedure of RBFNN, the sliding window slides along the sensitive element, and each prediction is conducted for the $3 \mathrm{D}$ force sensed by the sensitive ele- ment at the center of the sliding window. In the experiment, sliding windows of sizes 3,5 , and 7 are used to construct input vectors of the RBFNN, respectively, and to verify how the sliding window size influences the prediction results. As described in Section 3.2, 7 is the optimal spread value for the RBFNN prediction model; the prediction results of $3 \mathrm{D}$ force components based on different sliding windows with spread $=7$ of the RBFNN are shown in Table 3 . Table 3 shows the relative prediction errors of $F_{x}, F_{y}$, and $F_{z}$ loaded on the flexible tactile sensor, respectively. The testing samples used in Table 3 are the same as in Table 1.

As can be seen from Table 3, when the sliding window size is 3 and 5 , the performance of the RBFNN predictor is significantly improved and the prediction error is reduced in comparison with the results in Table 1. The prediction errors of $F_{x}, F_{y}$, and $F_{z}$ are significantly lower than those shown in Table 1 without the sliding window method, which means the characteristic information of sensitive elements close to the target element is properly extracted. That is very helpful and useful to improve the prediction accuracy and reduce the prediction error of the $3 \mathrm{D}$ force. The best prediction results for $F_{x}, F_{y}$, and $F_{z}$ are gained with the sliding window size of 5 , and the RBFNN reaches its best performance. However, as the sliding window continues to increase, the prediction error tends to increase and the accuracy tends to decrease. In the experiment, when the window size further increased, the information of the sensitive element which is far away from the target sensitive element is extracted. At that point, the correlation between the distant sensitive units 


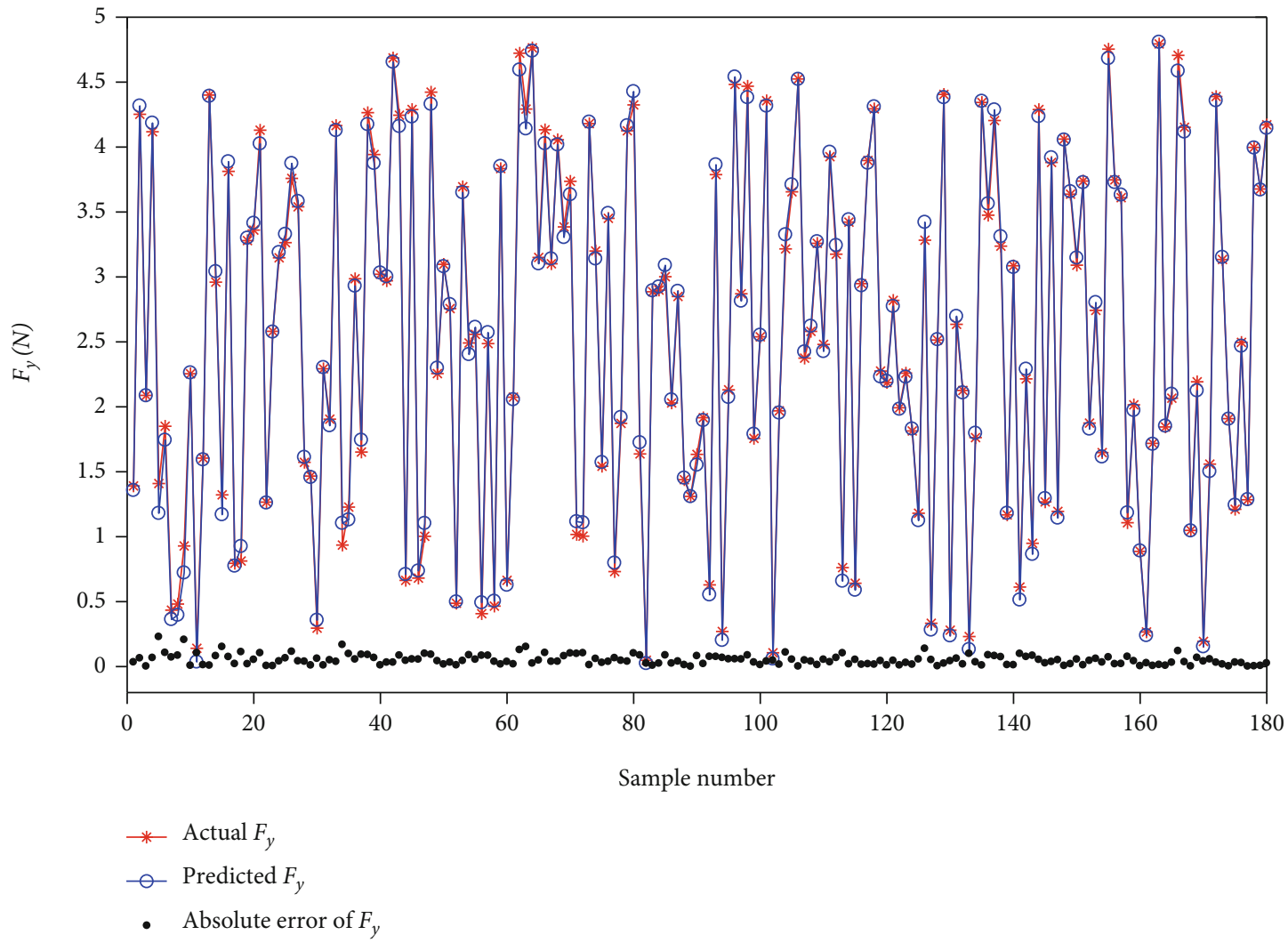

Figure 7: Prediction results of $F_{y}$ (the mean relative prediction error is $4.61 \%$ ).

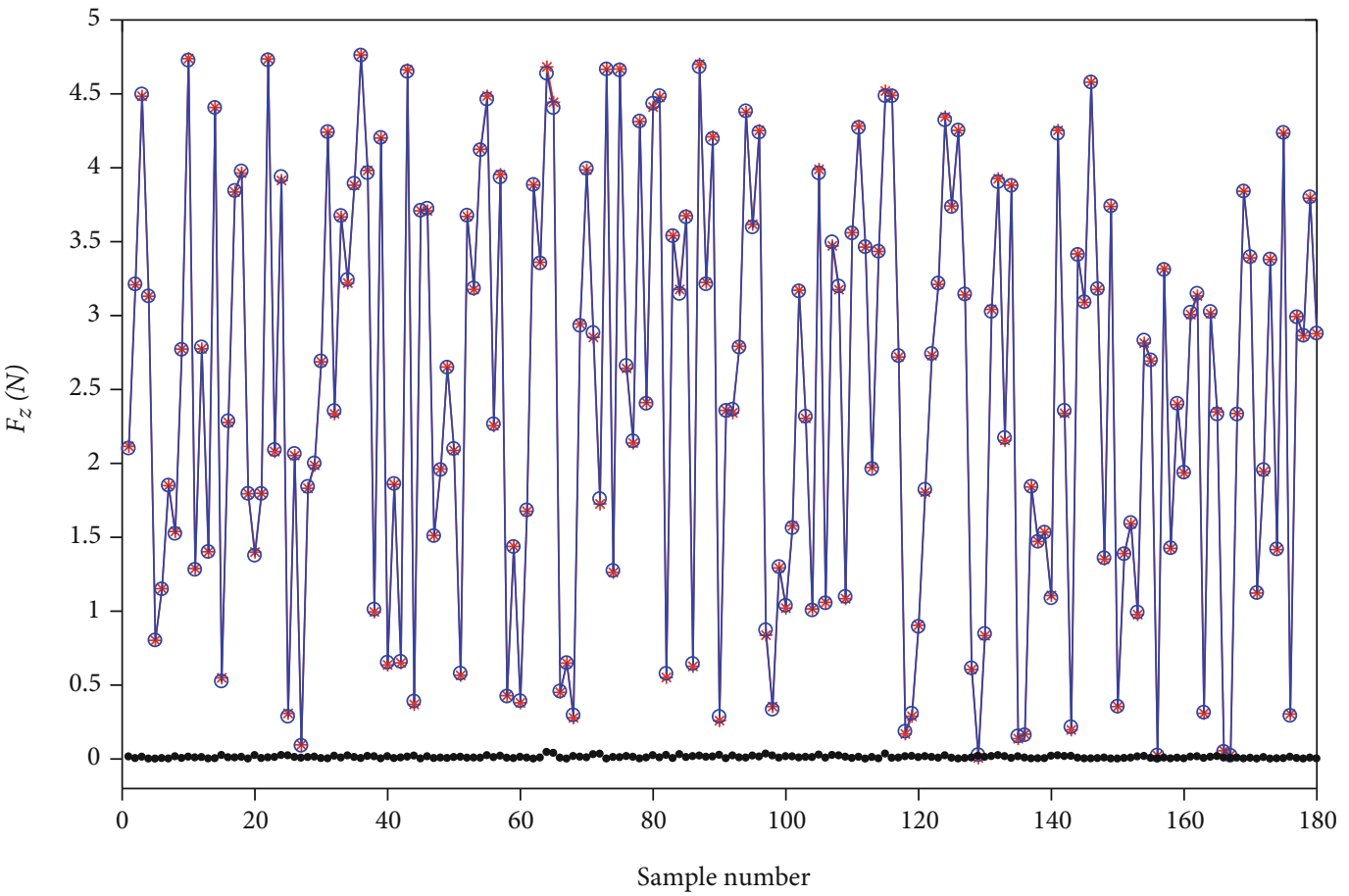

* Actual $F_{z}$

- Predicted $F_{z}$

- Absolute error of $F_{z}$

Figure 8: Prediction results of $F_{z}$ (the mean relative prediction error is $1.96 \%$ ). 


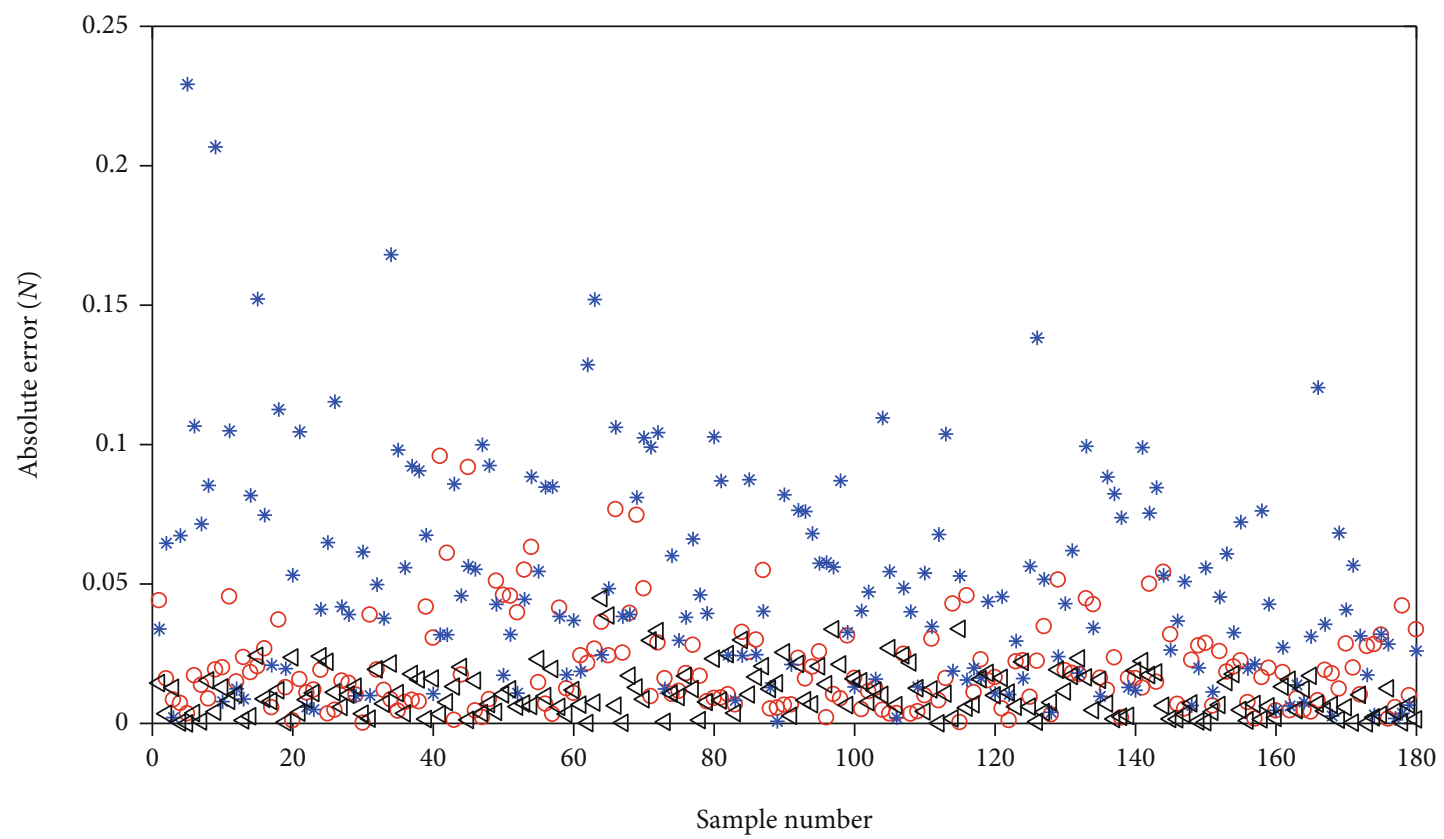

○ Absolute error of $F_{x}$

* Absolute error of $F_{y}$

$\triangleleft$ Absolute error of $F_{z}$

Figure 9: Absolute prediction errors of $F_{x}, F_{y}$, and $F_{z}$.

TABle 2: Absolute error of $F_{i}(i=x, y, z)$ force components.

\begin{tabular}{ccccc}
\hline $\begin{array}{c}\text { Minimum } \\
\text { absolute } \\
\text { error }(N)\end{array}$ & $\begin{array}{c}\text { Maximum } \\
\text { absolute } \\
\text { error }(N)\end{array}$ & $\begin{array}{c}\text { Mean } \\
\text { absolute } \\
\text { error }(N)\end{array}$ & $\begin{array}{c}\text { Standard } \\
\text { deviation of } \\
\text { the absolute } \\
\text { error }(N)\end{array}$ \\
\hline$F_{x} \quad 1.07 e-4$ & 0.0449 & 0.0203 & 0.0171 \\
$F_{y}$ & $7.45 e-4$ & 0.2292 & 0.0510 & 0.0393 \\
$F_{z}$ & $1.71 e-5$ & 0.0449 & 0.0108 & 0.0085 \\
\hline
\end{tabular}

All of the prediction results including absolute errors and relative errors prove that the optimal RBFNN model could be well exerted on 3D force prediction for the flexible tactile sensor and improve the prediction results.

TABLE 3: Prediction results of 3D force based on different sliding windows.

\begin{tabular}{lccc}
\hline & $E_{F x}$ & $E_{F y}$ & $E_{F z}$ \\
\hline Sliding window of size 3 & $2.53 \%$ & $3.05 \%$ & $1.83 \%$ \\
Sliding window of size 5 & $2.13 \%$ & $2.71 \%$ & $1.36 \%$ \\
Sliding window of size 7 & $2.76 \%$ & $3.62 \%$ & $1.84 \%$ \\
\hline
\end{tabular}

$E_{F i}$ denotes the mean relative prediction error of force component $F_{i}$ $(i=x, y, z)$ for 300 testing samples.

and the target sensitive unit becomes weak, and their characteristic information would interfere with each other, so it would reduce the prediction accuracy of the target sensitive element. Experimental results show that setting the size of the sliding window appropriately can effectively reduce the prediction error of $3 \mathrm{D}$ force, improve the approximation per-
TABLE 4: Comparison of prediction results.

\begin{tabular}{lccc}
\hline Prediction error & $E_{F x}$ & $E_{F y}$ & $E_{F z}$ \\
\hline With sliding windows (Win 5) & $2.13 \%$ & $2.71 \%$ & $1.36 \%$ \\
Without sliding windows & $2.62 \%$ & $3.44 \%$ & $1.82 \%$ \\
\hline
\end{tabular}

Win 5 means the size of the sliding window is 5 .

formance of RBFNN, and make the flexible tactile sensor more practical and feasible. The best prediction results for $3 \mathrm{D}$ force from Tables 1 and 3 are compared in Table 4 .

In Table 4 , all the prediction results are conducted by the RBFNN model whose spread is 7 . In the second line of Table 4, the prediction results based on sliding window technology are much better than those in the last line without using the sliding window method. It means that sliding window technology is very valid in $3 \mathrm{D}$ force prediction for the tactile sensor and plays an important role in it.

\section{Conclusions}

In this paper, the property and structure of the flexible tactile sensor are described, and the detecting principle of the sensor is analyzed. Based on the piezoresistive effect of the conductive rubber, the optimized RBFNNs have been used to achieve the accurate prediction of multiple $3 \mathrm{D}$ forces loaded on the surface of the flexible tactile sensor from different aspects. The $k$-means and RLS algorithms are used to optimize the RBFNN predictor. The optimized RBFNN constructed with different spreads and based on the sliding window technology has gained great results, decreased the 
prediction error of 3D force, and improved the prediction ability of the RBFNN model. All the results indicate that the RBFNN method can be efficiently applied to the 3D force prediction research of the flexible tactile sensor. For future work, we will focus on improving the properties of forcesensitive materials and the structure of sensitive elements and developing other machine learning algorithms to realize real-time prediction of 3D force for the tactile sensor so that it can accomplish better results under different environments and could be used as real robot skin.

\section{Data Availability}

The data used to support the study is available upon request to the corresponding author.

\section{Conflicts of Interest}

The authors declare that there is no conflict of interest regarding the publication of this paper.

\section{Acknowledgments}

This research was supported by the Nature Science Research Key Project for Universities in Anhui Province (KJ2019A0769), the Nature Science Foundation of Anhui Province (1908085MF204), the Key Outstanding Young Talents Support Project for Universities in Anhui Province (gxyqZD2018057 and gxyq2019056), the Quality Engineering Project for Universities in Anhui Province (2018jyxm0459, 2019zyrc081, and 2020szsfkc0294), and the Quality Engineering Project of Anhui Jianzhu University (2020szkc03 and 2018jy03).

\section{References}

[1] C. Shao, S. Tananka, T. Nakayama, Y. Hata, and T. Bartley, “A tactile sensor network system using a multiple sensor platform with a dedicated CMOS-LSI for robot applications," Sensors, vol. 17, no. 9, p. 1974, 2017.

[2] J. X. Liu, N. Liu, K. Hashimoto, C. Z. Meng, and S. J. Guo, "Touch position identification based on a flexible array-less super capacitive tactile sensor," AIP Advances, vol. 9, pp. 1-6, 2019.

[3] S. El-Molla, A. Albrecht, E. Cagatay et al., "Integration of a thin film PDMS-based capacitive sensor for tactile sensing in an electronic skin," Journal of Sensors, vol. 2016, 7 pages, 2016.

[4] G. Liang, Y. Wang, D. Mei, K. Xi, and Z. Chen, "Flexible capacitive tactile sensor array with truncated pyramids as dielectric layer for three-axis force measurement," Journal of Microelectromechanical Systems, vol. 24, no. 5, pp. 1510-1519, 2015.

[5] Y. Huang, H. Yuan, W. Kan, X. Guo, C. Liu, and P. Liu, "A flexible three-axial capacitive tactile sensor with multilayered dielectric for artificial skin applications," Microsystem Technologies, vol. 23, no. 6, pp. 1847-1852, 2017.

[6] I. M. Koc and E. Akca, "Design of a piezoelectric based tactile sensor with bio-inspired micro/nano- pillars," Tribology International, vol. 59, no. 3, pp. 321-331, 2013.

[7] H. K. Kim, S. Lee, and K. S. Yun, "Capacitive tactile sensor array for touch screen application," Sensors and Actuators A Physical, vol. 165, no. 1, pp. 2-7, 2011.
[8] T. Huang, S. Yang, P. He et al., "Phase-separation-induced PVDF/graphene coating on fabrics toward flexible piezoelectric sensors," ACS Applied Mater Interfaces, vol. 10, no. 36, pp. 30732-30740, 2018.

[9] B. Sümer, B. Aksak, K. Şsahin, K. Chuengsatiansup, and M. Sitti, "Piezoelectric polymer fiber arrays for tactile sensing applications," Sensor Letters, vol. 9, no. 2, pp. 457-463, 2011.

[10] Y. Zhang, J. Ye, Z. Lin, S. Huang, H. Wang, and H. Wu, “A piezoresistive tactile sensor for a large area employing neural network," Sensors, vol. 19, 2019.

[11] Y. Song, F. L. Wang, and Z. Y. Zhang, "Decoupling research of a novel three-dimensional force flexible tactile sensor based on an improved BP algorithm," Micromachines, vol. 9, no. 5, p. 236, 2018.

[12] L. Massari, E. Schena, C. Massaroni et al., "A machinelearning-based approach to solve both contact location and force in soft material tactile sensors," Soft Robotics, vol. 7, no. 4, pp. 409-420, 2020.

[13] G. Schwartz, B. C.-K. Tee, J. Mei et al., "Flexible polymer transistors with high pressure sensitivity for application in electronic skin and health monitoring," Nature Communications, vol. 4 , no. 1, 2013.

[14] M. Asadnia, A. G. P. Kottapalli, J. M. Miao, and M. S. Triantafyllou, "Ultra-sensitive and stretchable strain sensor based on piezoelectric polymeric nanofibers," in in Proceedings of the IEEE International Conference on MEMS, pp. 678-681, Estoril, Portuga, Jan. 18th-22th, 2015.

[15] H. K. Lee, J. Chuang, S. I. Chang, and E. Yoon, "Normal and shear force measurement using a flexible polymer tactile sensor with embedded multiple capacitors," Journal of Microelectromechanical Systems, vol. 17, no. 4, pp. 934-942, 2008.

[16] W. Liu, C. Gu, R. Zeng, P. Yu, and X. Fu, "A novel inverse solution of contact force based on a sparse tactile sensor array," Sensors, vol. 18, 2018.

[17] P. Mittendorer, E. Yoshida, and G. Cheng, "Realizing wholebody tactile interactions with a self-organizing, multi-modal artificial skin on a humanoid robot," Advanced Robotics, vol. 29, no. 1, pp. 51-67, 2015.

[18] A. Cirillo, P. Cirillo, G. D. Maria, C. Natale, and S. Pirozzi, "Modeling and calibration of a tactile sensor for robust grasping," Congress of the IFAC, vol. 50, no. 1, pp. 68436850, 2017.

[19] P. Liu, Y. Huang, C. Lian, H. S. Jiang, and Y. J. Ge, "Sensitive coefficient of pressure-sensitive sensor based on conductive rubber filled by carbon black," Acta Materiae Compositae Sinica, vol. 29, no. 1, pp. 16-20, 2012.

[20] Y. Huang, P. Liu, Y. G. Zhang, and Y. J. Ge, "Piezoresistive characteristics and its fitting method of conductive rubber for flexible tactile sensors," Journal of university of science and technology of China, vol. 4, no. 3, pp. 200-206, 2011.

[21] S. Xin, X. K. Zhuang, H. Q. Pang, and P. Shuang, "Numerical model of a novel tactile sensor based on finite element analysis," in in Proceedings of the IEEE International Conference on ROBIO, pp. 1973-1978, Guangzhou, China, Dec. 11th 14th, 2012.

[22] K. L. Johnson, Contact Mechanics, Cambridge University Press, Cambridge, NY, USA, 2012.

[23] W. Ma and E. Fan, "Linear superposition principle applying to Hirota bilinear equations," Computers and Mathematics with Applications, vol. 61, no. 4, pp. 950-959, 2011. 
[24] E. Hartman, J. D. Keeler, and J. M. Kowalski, "Layered neural networks with Gaussian hidden units as universal approximations," Neural Computation, vol. 2, no. 2, pp. 210-215, 1990.

[25] J. Park and L. W. Sandberg, "Universal approximation using radial-basis-function networks," Neural Computation, vol. 3, no. 2, pp. 246-257, 1991. 